\title{
Opening doors to discovery: Partnerships are key to advancing open science
}

\author{
Frank Vrancken Peeters* \\ CEO, Springer Nature, Berlin, Germany
}

\begin{abstract}
The evolution of scholarly communications has accelerated in recent years, and 2020 for obvious reasons put even more pressure on the sector to evolve and adapt. By opening up access to research publications, by simplifying or customising the digital experience, or by improving the speed of publishing - the focus is firmly placed on the need for publishers to work more in partnership with each other, with institutions, funders, and new players in the market to develop solutions that meet the evolving needs of researchers and the wider community. Partnerships between different actors in the research process address challenges in practice and help advance open science, publishing, and the research system as a whole.
\end{abstract}

\section{The pandemic has only made the case for 'open' stronger}

Open science is "the idea that scientific knowledge of all kinds should be openly shared as early as is practical in the discovery process." ${ }^{1}$ Open science brings huge advantages: It benefits research as well as society as a whole, as the past year has shown like no other.

This is not only about open access to publications, but about every element of the research process. Research data, protocols, positive and negative results - making all of these elements instantly available to all levels of an inquiring society, not just to the research community, can make research more relevant and effective. It can also drive efficiencies in the research process. The pandemic has demonstrated the power of everyone working together in the most effective way possible.

Research has achieved so much in response to the pandemic: Within one month of the first reported case of COVID-19, the virus was rapidly sequenced and openly posted to GenBank, the genetic sequence database of the US National Institutes of Health. Scores of researchers shared their early findings about COVID-19 as openly accessible preprints. These findings were tested and refined in real-time discussions that were tracked publicly and transparently. Research that could not withstand replication and reproducibility efforts was quickly and publicly debunked, allowing the scientific community to pursue more promising avenues. Publishers made subscription articles relevant to coronavirus freely available to all. All of this played a role in the incredibly fast progress that was made, including the development of vaccines.

From a societal point of view, the COVID-19 pandemic highlighted that the daily workings of science have practical ramifications in all our lives. Scientific discoveries affect not just researchers working in laboratories, but also policy makers, doctors, patients, families and the general public.

\footnotetext{
*E-mail: frank.vranckenpeeters@springernature.com.

${ }^{1}$ Michael Nielsen, Reinventing Discovery, Princeton University Press, 2011.
} 
Open science promotes transparent and accessible knowledge that is shared and developed through collaboration for the benefit of all, allowing more voices to join the scientific conversation. This brings benefits not only during emergencies, but makes research more relevant and effective and generally drives efficiencies in the research process.

\section{The long road to open science}

So how can open science be achieved? It has been a long road so far and though progress especially in the past few years has been very encouraging, there is still quite a way to go before the goal is reached.

Around 30 years ago, publishing was a fairly straightforward proposition. Publishers focused on manuscripts submitted by authors, and agents dealt with the distribution of print journals. In the next phase starting in the 1990s, publishers and libraries moved from print to digital. This transition enabled easier distribution and also individual and remote access to publications. The transition from subscriptions to the open access model began a decade later, and will continue for years to come. Apart from the obvious benefit of unrestricted access to research, it also rewards authors with significantly more visibility for their work. For publishers, business models which support open access scale with the number of articles and bring the value provided more in line with revenue - which should enhance trust and encourage investment.

The addition of enhanced research solutions has only begun recently and has huge potential to provide increased value to researchers and the wider community - such as better research assessment for funders, researchers, and research institutions; artificial intelligence support for language editing services for researchers; or specialist databases for institutions, to name but a few.

These foundational developments have already taken two decades and the transition to open science is still moving slowly. It is not just publishers that have to find a way forward to new publishing models and norms, there are also many researchers who are not incentivised to treat this as a priority, and many funders around the world who do not support the transition financially. So how can the transition move forward at greater speed?

\section{Speeding up the transition to open science}

Developments driven by the pandemic have shown that research and its publication can indeed move faster.

The urgency of the global health crisis promoted the speedy sharing of research results: Preprints were shared more often, clinical trials and peer review were expedited, data were shared more quickly, and funders allowed the diversion of funds to COVID-19 research. As a result, publication of research articles grew by $8 \%$ in 2020, and COVID-19 related papers contributed around $4 \%$ of this growth.

Publishers stepped up very quickly and more than 50,000 COVID-19 related papers were made freely available within weeks. In the first half of 2020, around 150 million people read or downloaded this research, making it one of the most sought-after topics of all time. ${ }^{2}$ Many publishers also continue to

\footnotetext{
${ }^{2}$ Research Information: 'Publishers swift to release Covid-19 research - STM Association', 1 July 2020.
} 
work with global organisations such as the World Health Organization to support the sharing of relevant research and data.

Publishers supported remote access to publications for researchers as universities were required to close, and for teachers and lecturers to ensure that children and students were able to continue with their studies when they were required to learn from home. This required considerable effort from publishers and industry initiatives ${ }^{3}$, but resulted in everyday benefits and efficiency gains visible in web visits and downloads - a clear sign that researchers and also the interested public relied on research publications during this time.

All of these developments demonstrated the very responsive nature of the publishing sector, and placed a spotlight on the importance of immediate access to research, data sharing and curation as well as good data management for boosting the reproducibility and reliability of research.

\section{Continued focus on quality and rigour}

While speedy sharing of research results drives further research advances, the quality and rigour of published research should not be compromised. Rigorous peer review needs to be ensured, and when research is communicated readers should be able to determine whether they are looking at a preprint, a work-in-progress article, or a final peer-reviewed publication.

Upholding these standards is important to contain the risk of misunderstandings and disinformation. The status of article versions of COVID-19 research led to confusion especially among non-expert audiences. Early sharing of preprints is a positive development, as it allows research findings to be communicated as early as possible among researchers. But to audiences outside of research, it is not always clear that preprints have not undergone peer review and need to be treated differently than a final published article, also known as the 'version of record'.

Accepted manuscripts have usually benefited from peer review but are still work-in-progress versions of research findings and not systematically maintained and corrected. Creating multiple unconnected versions of the research article, with growing numbers freely available online and some containing uncorrected errors, risks undermining trust in science.

Public trust in science is crucial at times of public health emergencies. It calls for the quality-assured, value-added final published article to be open in perpetuity, dynamically updated by editors and publishers who have a duty of care for the scientific record. For this version of record to be made freely accessible and re-usable, a sustainable publishing model is needed that secures the funding necessary to ensure the quality and integrity of the final article. The so-called 'Gold' open access model provides this, as researchers or their funders pay a publication fee to ensure sustainability.

The sharing of the work-in-progress accepted manuscript, on the other hand, also known as 'Green' open access, neither provides access to the quality-assured final article, nor does it offer a sustainable open access model, as it continues to rely on subscription revenue to pay for the added value. The increased attention that Green open access received in the past year risks misunderstandings about article versions, and at the same time threatens the sustainability of the transition.

The transition to open science needs to be advanced sustainably, with a continued focus on quality, and at the same time with greater speed.

\footnotetext{
${ }^{3}$ Such as SeamlessAccess and GetFTR.
} 


\section{Open to collaboration: Partnerships can advance open science}

The speedy reactions during the pandemic show how much can be achieved when working in partnership. All actors in the research landscape - individual researchers and editors, libraries, funders, institutions, publishers and new players - need to work together to move open science forward and achieve efficiency and effectiveness for the research process.

Lofty declarations and good intentions are not enough. It is positive that the concept of open science receives attention even at a level such as the $\mathrm{G} 7^{4}$, but it needs practical approaches and detail work to actually move forward and achieve tangible results.

All actors in the research process, including publishers, need to be open for new solutions and prepared to experiment with each other, with researchers and the wider community, including new players in the market. There are numerous examples of such partnerships which support the transition to open science.

\section{Partnership 1: Transformative agreements enable large scale transition to open access}

Transformative agreements are one example of libraries, consortia, institutions and funders working with publishers to move more quickly towards open access. Many transformative agreements are great examples of partnership - they show that remarkable progress can be achieved in a relatively short time by working together across the research landscape.

There are two key reasons why these agreements work well: First, they provide a framework for libraries or institutions to channel budgets so far used for subscriptions into open access publishing. This is important because changing budget streams is one of the major complexities in making open access happen. Second, they are so successful because they make open access much easier for researchers as they do not have to come up with funding for open access publishing fees (also known as APCs) individually. This is crucial because the availability of funding varies considerably between disciplines, and researchers in some subjects - such as the humanities - often find it more difficult to cover APCs.

There is no standard model, no blueprint available for transformative agreements because the research landscape varies from country to country. This means it is all the more important to be open to new solutions and also invest time and resources into these partnerships: Negotiations can take a lot of time, it is important to build trust and the detailed work necessary is easy to underestimate. But the result can be mutually beneficial, and once in place can bring open access growth very quickly.

For example, the transformative agreement with Projekt DEAL in Germany not only provides full access to Springer Nature content, it also enables all researchers to publish open access irrespective of their research field - and therefore increases equity. Comparing the first half of 2019 with the first half of 2020, the open access share of Springer Nature publications in the humanities and social sciences in Germany went from $10 \%$ to $71 \%$.

Another example, the transformative agreement with the University of California provides a new flexible multi-payer model allowing both library and funder to contribute to open access publishing. This was necessary for the different funding landscape in the US and required investment in a purpose-built technological solution to support it. From 2021, the University of California will cover the first US\$1,000

\footnotetext{
${ }^{4}$ Carbis Bay G7 Summit Communiqué: https://www.whitehouse.gov/briefing-room/statements-releases/2021/06/13/carbisbay-g7-summit-communique/.
} 
of each APC, and the remainder due will be covered either by the authors' individual research funds, or by UC when such funds are unavailable.

The results speak for themselves: Recent data shows that across the eight countries where Springer Nature had a national transformative agreement live in 2019 , between $70 \%$ and $90 \%$ of content was published immediately open access. ${ }^{5}$

\section{Partnership 2: Making articles accessible where researchers want to read them}

Publishers are also open to partnerships that make the research process more efficient and effective. Content syndication is one tool to support this as it provides publications directly or indirectly to researchers on platforms where they gather and work together.

Partnering with ResearchGate, a popular platform with researchers, improves the user experience for readers of Springer Nature content and drives usage for authors. The partnership creates a seamless experience for readers, and $90 \%$ of surveyed researchers were positive about accessing the version of record more easily ${ }^{6}$. This partnership brought together two organisations with differing areas of expertise but similar goals.

In another example of collaboration, publishers have worked with SeamlessAccess to address the sometimes confusing and frustrating experience researchers were facing when they were trying to access publications subscribed to by their university from outside the institution's network. The new solution means that users from participating institutions only have to log in once per browser and their institutional affiliation is remembered, making future authentication easier. This model was implemented just in time to facilitate the tremendous increase in remote working in 2020.

\section{Partnership 3: The societal impact of open science}

Open science is fundamentally changing the way that researchers communicate and collaborate. Dynamic workflows are emerging, and distribution channels are changing, enabling others - such as patients, businesses, teachers, policy makers - to benefit from critical insights. This has increased the societal impact of open research and its relevance.

The 17 United Nations Sustainable Development Goals (SDGs) prioritise the most important changes we all must make. Science and research are key in understanding their impact, and in finding and delivering solutions. But progress on these goals lags far behind - COVID-19 has challenged society, and in some cases has exposed the gaps between the latest scientific insights and policy-making. Collaboration is all the more important if we are to make progress on sustainability, and interdisciplinary thinking is needed to address the world's greatest challenges.

A joint project between Springer Nature, the Association of Universities in the Netherlands (VSNU) and the Dutch University Libraries and the National Library Consortium (UKB) explored how research content is being used outside of academia. Bibliometric analysis of nearly 360,000 SDG-related documents published in 2017 and a survey of nearly 6,000 readers on Springer Nature websites was summarised in

\footnotetext{
${ }^{5}$ Springer Nature's Transformative Agreements enable country level transition to open access.

${ }^{6}$ Springer Nature and ResearchGate to move forward with long-term content-sharing partnership.
} 
the white paper Open for All, Exploring the Reach of Open Access Content to Non-Academic Audiences ${ }^{7}$. The results show not only a notable open access advantage for usage and attention of SDG publications: Documents published immediately open access have on average 4.4 times as many downloads and are shared more often. More importantly, the results also demonstrated who that research is reaching, and a significant benefit of open access to non-academic audiences: From the reader survey, about $40 \%$ of readers were classified as user groups outside of academia.

\section{The contribution of publishing to the research process}

These examples show what constructive and practical partnerships can achieve for the research process. Sharing experience and data points is important to earn the trust of the various actors involved.

This kind of tangible progress matters so much because Research \& Development is tremendously important, as the past year has shown like no other. Global R\&D spending has reached almost US\$1.7 trillion. ${ }^{8}$ Publishing is only a small element of the R\&D process in terms of expenditure - it costs less than $1 \%$ of R\&D spending - but it has a big role to play in helping to ensure that the other $99 \%$ is used more efficiently and effectively to accelerate progress.

This potential is real and quantifiable. For example, a PwC study for the EU Commission estimates the cost of not having FAIR research data at up to $€ 26$ billion in Europe alone. ${ }^{9}$ FAIR means "findable, accessible, interoperable, re-usable", so this estimate includes the cost of being unable to access and reuse data from research work done in Europe, and of duplicated work due to a lack of awareness of research or unpublished negative results. To put this into perspective, $€ 26$ billion is around 2.5 times the annual global spend on journal publishing. ${ }^{10}$ The benefits of open science and increased efficiency to the research system are huge compared with current spending on publishing.

The speed of publication is another area that could bring tremendous benefits. If time to publication for every research article could be reduced by one-fourth, or even one-third, this would bring enormous gains - not only to the research process but to society as a whole, as the response to emergencies such as the pandemic shows particularly well. Openness is a key tool for progress and this includes enhancing diversity and inclusion in the research process itself.

For publishers, this is an exciting time, as it brings an opportunity to contribute more throughout the research process. Publishers may be a relatively small cog in terms of R\&D spending, but helping to open up science and to improve the efficiency of the research process means their contribution can be greater than it has been in the past.

Openness and transparency are necessary to gain trust. Trust is a basis for every partnership. And partnership and open science is the prize for us all as it will lead to an accelerated and more effective research system that delivers benefits for the whole world.

\footnotetext{
${ }^{7}$ White paper in partnership with VSNU, UKB and Springer Nature.

${ }^{8}$ UNESCO Institute for Statistics.

${ }^{9}$ Cost of not having FAIR research data, March 2018.

${ }^{10}$ The STM Report, 6th Edition, October 2018.
} 